\title{
Anthropomorphic Al Agent Mediated Multimodal Interactions in Vehicles
}

\author{
Satoshi Okamoto \\ Toyota Innovation Hub \\ San Francisco, U.S.A. \\ Satoshi_okamoto@calty.toyota.com \\ Shin Sano \\ Institute for Creative Integration \\ San Francisco, U.S.A. \\ ssano@creative-integration.com
}

AutomotiveUI '17 Adjunct, September 24-27, 2017, Oldenburg, Germany C 2017 Copyright is held by the owner/author(s).

\begin{tabular}{l} 
ACM ISBN 978-1-4503-5151-5/17/09. \\
https://doi.org/10.1145/3131726.3131736 \\
\hline
\end{tabular}

\begin{abstract}
This paper introduces a user experience design project that aspires to redefine the relationship between humans and vehicles. The authors have designed the visual representation of an artificial intelligence (AI) agent, Yui. The agent is intended to elicit a sense of collaboration with the vehicle, and enable to build rapport between driver and vehicle. In the age of automated vehicles (AVs) it is critical to develop such a cooperative relationship between humans and vehicles in order for the people to more readily adopt AVs. Autonomous capability is expected to roll out in progressive phases. In the phases of partially or conditional AVs, drivers need to be engaged and alert at all time so they can quickly take back control of the vehicle. This project attempts to utilize speculative prototypes of an anthropomorphic AI agent to explore the factors necessary to engage drivers in safe collaboration with AVs.
\end{abstract}

\section{Author Keywords}

Multimodal interactions; human agent interactions; user experience design; anthropomorphism

\section{CCS Concepts}

- General and reference Design - Information systems Multimedia information 


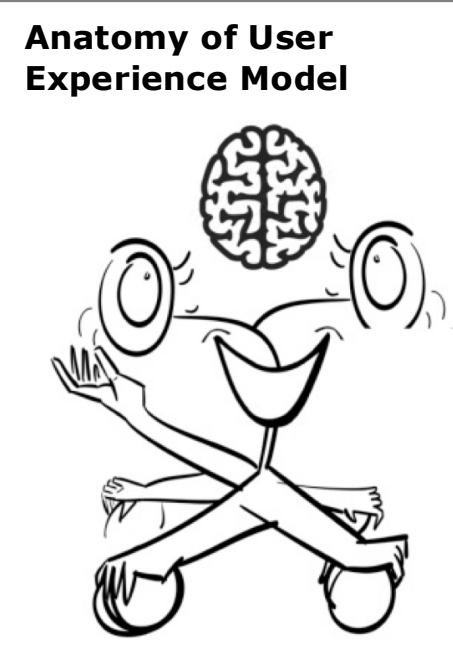

Figure 1: This image shows a metaphorical illustration of the comprehensive interaction system mediated by the AI agent, Yui, that enables seamless user experiences. The visual representation of the agent was initially called "Face", and sits in the center of the dashboard. The authors' group later developed the concept of Yui being the center of attention and traveling inside/outside the vehicle. It sees, listens to and speaks to the driver to understand, protect and delight him.

\section{systems - Human-centered}

computing Graphical user interfaces • Human-

centered computing Interaction design theory,

concepts and paradigms • Computing

methodologies Intelligent agents

\section{Objectives}

The authors' group designed the model of a set of multimodal interactions orchestrated by an anthropomorphized artificial intelligent (AI) agent, "Yui" to realize the following objectives (see Figure 1):

1. To understand the driver's state and intention

2. To offer safety and peace of mind to the driver

3. To engage the driver and the passengers in fun experiences

\section{Introduction}

AI enabled conversational agents (CAs) are increasingly being adopted to our everyday life [6]. Automotive original equipment manufacturers and their suppliers also have developed many voice-activated CA features on car infotainment systems with an emphasis on safety in recent years. Many of them, however, have received extensive criticism from consumers for glitches in their systems and have difficulty retaining the users engaged to actively use them. While the system is merely regarded as an instrument for the users most people are disappointed by the agent and stop interacting with it when it doesn't work the way they expect it to [4].

In the coming age of AVs, it is crucial that the CA system not only responds reliably upon the driver's commands merely as an instrument, but also that the system collaborates with the driver. For example, supporting the driver's decisions based on traffic and driver's state, flexible turn taking while communicating the intention of the action, generating a conversation that awakens the driver when s/he is drowsy or even inviting the driver to a location where a dramatic serendipity happens are highly collaborative and anticipating tasks. According Breazeal et al. [1], human-style cooperative behavior is an ongoing process of maintaining mutual beliefs, sharing relevant knowledge, coordinating action, and demonstrating commitment to doing one's own part, helping the other to do theirs, and completing the shared task.

Authors designed the agent, Yui aspiring to create the above mentioned collaborative relationship between drivers and vehicles. It is our attempt to move beyond CAs as tools or instruments to CAs that interact with drivers as capable and cooperative partners. The relationship should mirror those between close friends or family members who share a common purpose, sometimes watching over and helping each other out.

\section{Features that Yui mediates}

Yui manages the functions that are non-existing, but speculated to be available with current and emerging technologies. To understand the driver, Yui detects the driver's status instantaneously, capturing emotions, stress levels, degree of fatigue and arousal state. The system also accumulates information about drivers' preferences, such as points of interest, food, music, travel styles and even a potential affection for someone from meta-analytics of social media activities and conversation logs. Based on this data the system generates multi-sensory inputs to keep the driver engaged and alert.

To offer safety and peace of mind Yui pays attention to the outside environments utilizing comprehensive traffic recognition sensor systems. Automated driving is 


\section{Prescriptive scenario}

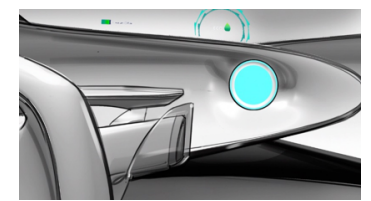

Figure 2. Default conversation mode of Yui

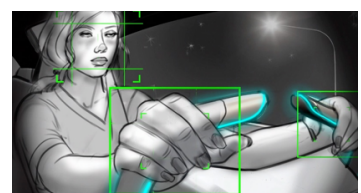

Figure 3. The system detects that the driver is drowzy

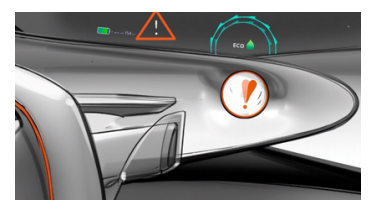

Figure 4. Yui morphs into an exclamation mark to alert the driver

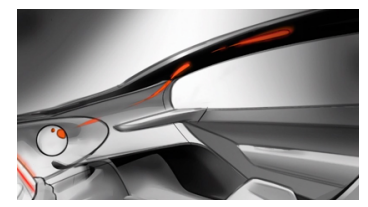

Figure 5. Yui travels to draw the driver's attention to the spot

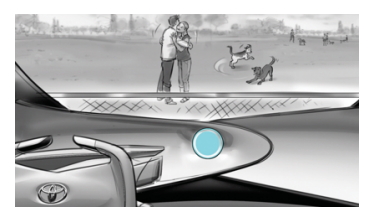

Figure 6. Yui invites you to pleasant surprise activated momentarily when Yui determines it is necessary to avoid a crash that is otherwise unavoidable by the human driver. Yui also suggests taking over driving when it detects the driver has a high degree of stress or fatigue. Yui travels on the vehicle's interior surfaces, drawing the driver's attention to areas where a potential danger is predicted, and gives the driver a tactile stimulus, as well as visual and auditory alerts. To engage the driver and passengers in an emotional experience Yui generates an "Emotion Map" which visualizes the locations and routes where the driver's notable emotions were detected by the computer vision. Yui also engages the driver in natural conversations based on his/her personal interests and even shows empathy to the drivers when $\mathrm{s} /$ he is feeling down. Yui even makes a thoughtful suggestion on a driving route to soothe him/her or on a destination where Yui knows the driver would come across with her/his sweetheart. The authors developed use case scenarios to describe the collaborative relationship between humans and vehicles, as well as how Yui watches over, supports and cares the driver through the features described above (see Figure 2-6).

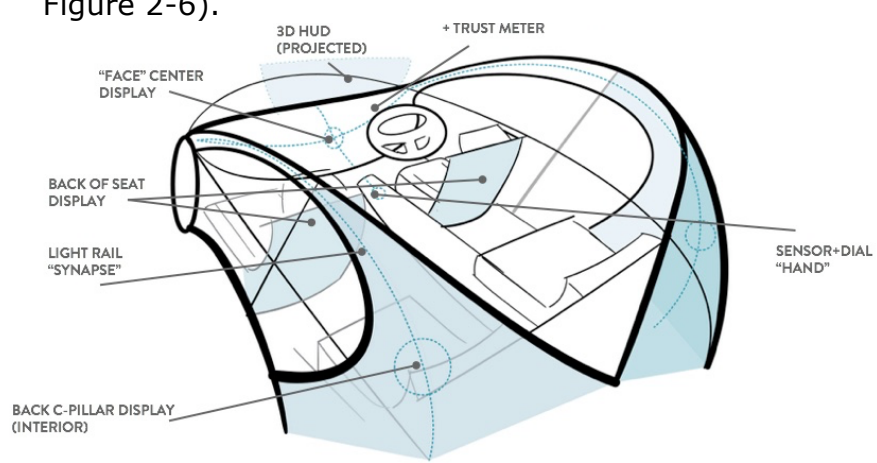

figure 7. The diagram shows the overall interaction deployment

\section{UX Design Principles and Execution}

The authors have established the following design principles of Yui-mediated user experiences (UX):

1. Yui is alive and mirrors human communication It is an embodiment of a vehicle, not a vehicle with a separate software system.

\section{Yui knows you and induces serendipity}

It uses its knowledge of the driver over time to anticipate needs and presents only relevant information at the time of need. It offers pleasant surprises by coordinating relevant events.

\section{Yui communicates to multiple senses}

It provides appropriate multi-sensory input to shorten the response time to hazardous situations and enhance pleasant experiences.

The authors explored literature on human robotic interactions and human agent interactions field to obtain theoretical frameworks on designing Yui. Media equation theory [8] shows that people tend to treat computers and other media as if they were rea people. Yee et al. [11] found a visual representation of an agent leads to more positive social interaction than not having a visual representation but, the realism of the embodied agent may matter very little. Hoffman et al. [3] proposed movement-centric design in human robotic interactions, which suggests sophistication in the way robots move instead of in the way they look. Breazeal et al. [2] suggests the importance of nonverbal communications, such as gesture, especially in coordinating teammate' actions for collaborative activities.

With these studies in mind the authors avoided human facial features such as eyeballs, as well as cold, technical manner of graphic treatment (see Figure 9). 


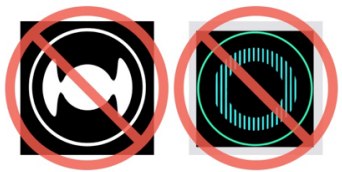

No cold tech feeling

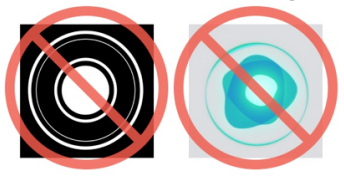

No eye-ball like shape

Figure 9: "Don'ts" for the Yui visual representation.

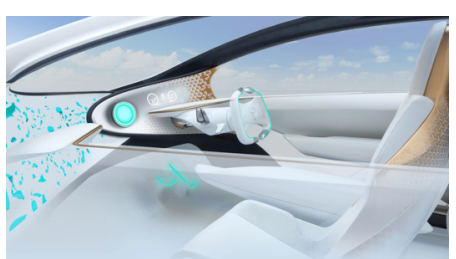

Figure 10. Interior of the prototype vehicle.

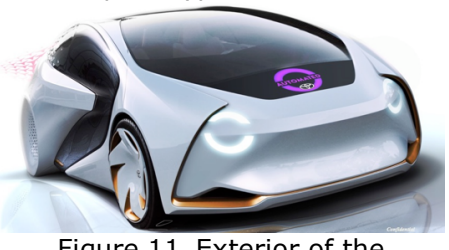

Figure 11. Exterior of the prototype vehicle.

Figure 13(Right). A motion graphic shows abstract leaves being blown, coordinated with sound effects and wind to awaken the driver.
Instead the authors maintained a rather abstract figure of the agent and employed anthropomorphism to introduce attributes of human characteristics and qualities in gestures and behaviors as shown in Figure 8.

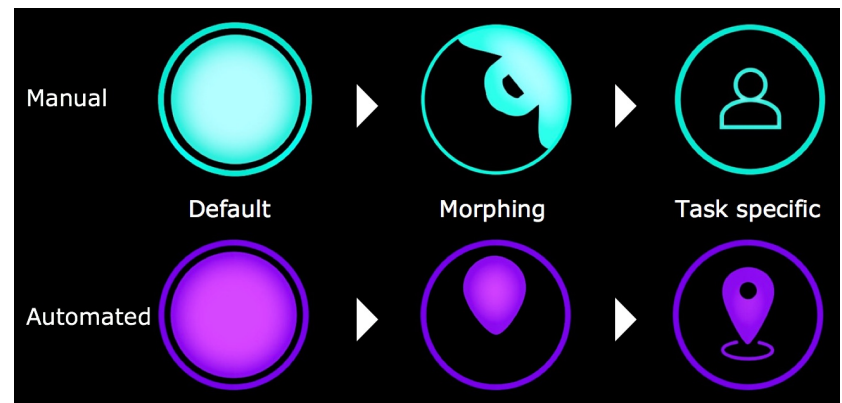

Figure 8. Visual representation of Yui. An abstract blob-like shape morphs to a task specific icon as necessary. The system status (manual or automated) is coded by different colors. The "Squash \& Stretch" animation gives a sense of speed, momentum, mass and weight that make Yui a living object.

For instance, "Squash \& Stretch", "Anticipation", "Slow in Slow out" as well as "Secondary Actions" techniques, introduced by Disney animators, Frank Thomas and Ollie Johnston, were utilized to create a personable and emotional appeal [10].

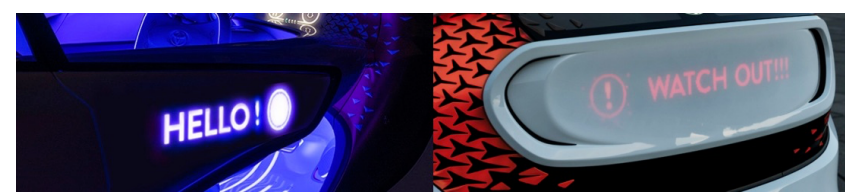

Figure 12 (Right). Yui travels outside of the vehicle to welcome the driver and alert pedestrians.

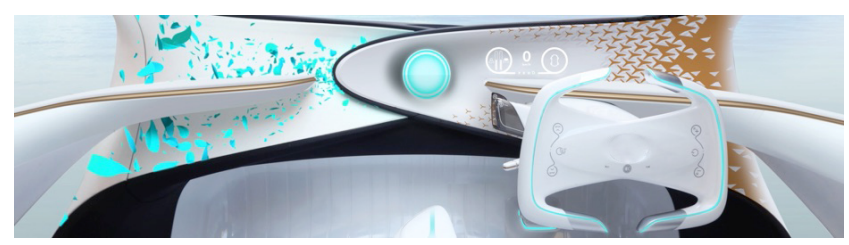

Implications and Future Research Suggestions The design concept introduced at this point are based on the designers' creative speculations with some literature studies. Many potential design factors and their parameters have not yet been examined and validated. Meanwhile, human factors in AVs have recently been studied with respect to the trust issue (Lee et al., 2016) [5], reflecting the driver's personality in automated systems (Sikkenk et al., 2015) [9], and exploring multimodal handover prompts (Politis et al., 2015) [7]. The authors see the potential to study these areas in conjunction with the human agent interactions. It is the authors' intention to inspire the Auto UI research community with this design concept and provide topics for future research, including:

- Can AI agent mediated interactions improve the adoption rate of AVs? How are they different between geographic and cultural profiles?

- What is the appropriate level of abstraction and degree of anthropomorphism in a visual representation of an AI agent?

- What is the best balance of verbal and non-verbal communication between humans and computer systems in the context of driving/riding AVs?

- How can AI agents facilitate communication between drivers in different AVs to enhance the experience?

\section{Acknowledgement}

This work was supported by Toyota Motor Corp.as a part of its concept vehicle development. The vehicle was displayed in public at 2016 CES in Las Vegas, NV, U.S.A. 


\section{References}

1. Breazeal, S., Brooks, A., Chilongo, D., Gray, J.,Huffman, G., Kidd, C., Lee, H., Lieberman, J., Lockerd, A. (2014). Working Collaboratively with Humanoid Robots. Proceedings of Humanoid Robots, 2004 4th IEEE/RAS International Conference on, Volume: 1

2. Breazeal, C., Kidd, C.D., Thomaz, A.L., Hoffman, G., Berlin, M. Effects of Nonverbal Communication on Efficiency and Robustness in Human-Robot Teamwork. Proceedings of 2005 IEEE/RSJ

International Conference on Intelligent Robots and Systems

3. Hoffman, G., Ju, W. (2014). Designing Robots with Movement in Mind. Journal of Human-Robot Interaction, Vol. 3, No. 1, 2014, Pages 89-122

4. Komatsu, T., Yamada, S. (2011). Adaptation Gap Hypothesis: How Difference Between Users'

Expected and Perceived Agent Functions Affect Their Subjective Impression. Systemics,

Cybernetics and Informatics, Volume 9 - Number 1 Year 2011. pp.67-74

5. Lee, J., Kim, N., Imm, C.., Kim, B., Yi, K. \& Kim, J. (2016) A Question of Trust: An Ethnographic Study of Automated Cars on Real Roads. Proceedings of Automotive UI '16. ACM, Ann Arbor, MI, USA, 201-208.

6. Parks Associates. "Voice Assistants and Technologies: Ecosystem and Market Leaders". http://www.parksassociates.com/blog/article/pr03212017 (accessed July 9, 2017)

7. Politis, I., Brewster, S. \& Pollick, F. LanguageBased Multimodal Displays for the Handover of
Control in Autonomous Cars. Proceedings of Automotive UI '15. ACM, Nottingham, UK, 19-22.

8. Reeves, B., Nass, C. (1996). The Media Equation: How People Treat Computers, Television, and New Media Like Real People and Places. Cambridge University Press, pp.19-36

9. Sikkenk, M. \& Terken, J. Rules of Conduct for Autonomous Vehicles. Proceedings of Automotive UI '15. ACM, Nottingham, UK, 19-22.

10. Thomas, Frank; Ollie Johnston (1997) [1981]. The Illusion of Life: Disney Animation. Hyperion. pp. 47-69

11. Yee, N., Bailenson, J.N., Rickertsen, K. A MetaAnalysis of the Impact of the Inclusion and Realism of Human-Like Faces on User Experiences in Interfaces. Proceedings of CHI 2007, San Jose, CA, USA, 1-10 